\title{
Effect of the source charge on charged-boson interferometry
}

\author{
T. D. Shoppa, ${ }^{1}$ S. E. Koonin, ${ }^{2}$ and R. Seki ${ }^{2,3}$ \\ ${ }^{1}$ TRIUMF, Vancouver, British Colombia, Canada V6T $2 A 3$ \\ ${ }^{2}$ W. K. Kellogg Radiation Laboratory, 106-38 California Institute of Technology, Pasadena, California 91125 \\ ${ }^{3}$ Department of Physics and Astronomy, California State University, Northridge, California 91330 \\ (Received 19 November 1998; revised manuscript received 8 July 1999; published 5 April 2000)
}

\begin{abstract}
We investigate quantal perturbations of the interferometric correlations of charged bosons by the Coulomb field of an instantaneous, charged source. The source charge increases the apparent source size by weakening the correlation at nonzero relative momenta. The effect is strongest for pairs with a small total momentum and is stronger for kaons than for pions of the same momenta. The low-energy data currently available are well described by this effect. A simple expression is proposed to account for the effect.
\end{abstract}

PACS number(s): $25.75 . \mathrm{Gz}$

Intensity interferometry of charged particles from heavyion collisions is an important probe of the fireball created in such collisions. The source parameters (lifetime, size, etc.) are important in determining the energy density of the source, from which the equation of state can be extracted [1]. Unfortunately, the charged particles suffer Coulomb and strong interactions with each other and also with their source, complicating the interpretation of the interference patterns observed. The approximate Gamow correction for the Coulomb interaction between detected pions works well in the limit of the small source size [2], and improved corrections have been proposed for extended sources [3-6]. The effect of strong interactions between the two emitted particles on their correlations has been examined for two emitted pions or kaons [7,1], for a pair of the pion and kaon [9], and for the case of two emitted nucleons [10,7].

These calculations ignore the interactions of the emitted particles with the source. The effect of strong interactions with a source was investigated for pion-source absorptive optical potentials by Chu et al. [11] using eikonal wave functions. Because of its long range and the large charge of the colliding ions, the Coulomb influence of the source is also expected to be significant especially in low-energy collisions, and has been receiving much attention by the use of various approaches $[12-16,8]$. In this paper, we present a simple quantum-mechanical investigation, which we believe to clarify physics involved in the effect of the Coulomb interaction between the emitted particles and their source in low-energy collisions. For clarity, we examine two-boson interferometry of instantaneous sources. We find that the Coulomb interaction tends to increase the apparent source size, as it was found in Ref. [16], and that this apparent "magnification" can be evident in the experimental correlations binned by the total momentum of the pair.

The correlation between a pair of emitted particles is expressed in terms of their asymptotic momenta, $\mathbf{p}_{1}$ and $\mathbf{p}_{2}$. The correlation function depends on various factors: the symmetries between the emitted particles, the forces among the emitted particles and the source, and the size and lifetime of the source. Here, we treat the source massive; $\mathbf{p}_{1}$ and $\mathbf{p}_{2}$ are thus measured in the rest frame of the source, which does not recoil. The correlation function is expressed as

$$
C\left(\mathbf{p}_{1}, \mathbf{p}_{2}\right)=\frac{\int d \mathbf{r}_{1} d \mathbf{r}_{2} g\left(\mathbf{r}_{1}\right) g\left(\mathbf{r}_{2}\right)\left|\Psi_{\mathbf{p}_{1}, \mathbf{p}_{2}}^{(-)}\left(\mathbf{r}_{1}, \mathbf{r}_{2}\right)\right|^{2}}{\int d \mathbf{r}_{1} g\left(\mathbf{r}_{1}\right)\left|\phi_{\mathbf{p}_{1}}^{(-)}\left(\mathbf{r}_{1}\right)\right|^{2} \int d \mathbf{r}_{2} g\left(\mathbf{r}_{2}\right)\left|\phi_{\mathbf{p}_{2}}^{(-)}\left(\mathbf{r}_{2}\right)\right|^{2}},
$$

where the bosons are assumed to be emitted without correlation from the source with a momentum-independent semiclassical probability $g(\mathbf{r}) . \phi_{\mathbf{p}}^{(-)}(\mathbf{r})$ is the one-body scattering wave function of a boson with asymptotic momentum $\mathbf{p}$ and incoming scattered wave, due to the interaction with the source. $\Psi_{\mathbf{p}_{1}, \mathbf{p}_{2}}^{(-)}\left(\mathbf{r}_{1}, \mathbf{r}_{2}\right)$ is the two-body scattering wave function of the bosons with the asymptotic momenta $\mathbf{p}_{1}$ and $\mathbf{p}_{2}$.

To clarify the effect of the source-pair interaction, we neglect the Coulomb and strong interactions between the emitted pair, assuming that these can be treated by the methods in Refs. [2-4]. $\Psi_{\mathbf{p}_{1}, \mathbf{p}_{2}}^{(-)}\left(\mathbf{r}_{1}, \mathbf{r}_{2}\right)$ is then expressed as a symmetrized product of $\phi_{\mathbf{p}}^{(-)}(\mathbf{r})$ 's,

$$
\begin{aligned}
\Psi_{\mathbf{p}_{1}, \mathbf{p}_{2}}^{(-)}\left(\mathbf{r}_{1}, \mathbf{r}_{2}\right)= & 2^{-1 / 2}\left[\phi_{\mathbf{p}_{1}}^{(-)}\left(\mathbf{r}_{1}\right) \phi_{\mathbf{p}_{2}}^{(-)}\left(\mathbf{r}_{2}\right)\right. \\
& \left.+\phi_{\mathbf{p}_{1}}^{(-)}\left(\mathbf{r}_{2}\right) \phi_{\mathbf{p}_{2}}^{(-)}\left(\mathbf{r}_{1}\right)\right] .
\end{aligned}
$$

Note that Eqs. (1) and (2) give $C\left(\mathbf{p}_{1}, \mathbf{p}_{2}\right)=2$ at relative momentum, $\mathbf{q} \equiv \mathbf{p}_{1}-\mathbf{p}_{2}=0$, as is the case of no final state interaction with source. For further clarity, we take the source charge distribution to be Gaussian and define the apparent source size $R_{\text {app }}$ as

$$
\left.\frac{d C(q)}{d q^{2}}\right|_{q=0}=-\frac{1}{2} R_{\text {app }}^{2} .
$$

In general, $R_{\text {app }}$ will also depend upon the total momentum of the emitted pair, $\mathbf{P}=\mathbf{p}_{1}+\mathbf{p}_{2}$. Note that $R_{\text {app }}$ as defined becomes the exact source size, $R_{0}$, when there is no sourcepair interaction.

With no further approximation, we solve the Schrödinger equation numerically for $\phi_{\mathbf{p}}^{(-)}(\mathbf{r})$, using the partial-wave decomposition method with the appropriate kinematics and boundary conditions. Figure 1 shows $R_{\text {app }}$ thus calculated, illustrating the effect of a source charge on the correlations of both positive and negative pion pairs. We see that in both 


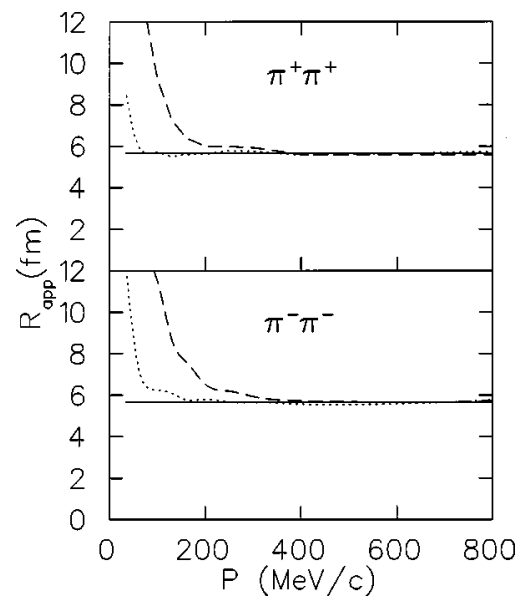

FIG. 1. The apparent source size $R_{\text {app }}$ as a function of the total momentum $P$ of $\pi^{+}$pairs (upper panel) and of $\pi^{-}$pairs (lower panel) emitted from a source of size $R_{0}=5.7 \mathrm{fm}$. $R_{\text {app }}$ is computed by a numerical integration of the Schrödinger equation. The dotted lines are the $R_{\text {app }}$ for the source charge $Z=15$, and the dashed lines for $Z=100$. The solid straight lines show $R_{\text {app }}=R_{0}=5.7 \mathrm{fm}$ for $Z=0$.

cases, the apparent size is larger than the exact size for small $P=|\mathbf{P}|$, as it was found in Ref. [16]. Also, as in Refs. $[13,16]$, the apparent size can become either larger or smaller than the exact size, depending on the charge of the pair being either negative or positive. It does, however, only by a very slight amount and at high $P$. We make two further comments on Fig. 1. (1) At a fixed $P, C(q)$ is of a Gaussian form as a function of $q$. (2) When $R_{\text {app }}$ is integrated over $\mathbf{P}$ with the thermal spectrum of pions, the resultant effective $R_{\text {app }}(T)$ is greater than $R_{0}$ (except for $\pi^{+}$pairs at high temperatures, $k T>400 \mathrm{MeV}$.)

A simplified description using the eikonal approximation reveals the essential physics of why the apparent size is larger than the true size. It provides a useful expression for the apparent size as a function of $P$. In the eikonal approximation

$$
\phi_{\mathbf{p}}^{(-)}(\mathbf{r})=\exp [i \mathbf{p} \cdot \mathbf{r}+i \chi(\mathbf{p}, \mathbf{r})]
$$

with

$$
\chi(\mathbf{p}, \mathbf{r})=-\frac{1}{v} \int_{\infty}^{0} V(\mathbf{r}+z \hat{\mathbf{p}}) d z,
$$

where $V(\mathbf{r})$ is the Coulomb potential between the source and the emitted boson of the mass $m$ and the velocity $v$. Equation (2) yields

$$
\begin{aligned}
\left|\Psi\left(\mathbf{p}_{1}, \mathbf{p}_{2}, \mathbf{r}_{1}, \mathbf{r}_{2}\right)\right|^{2}= & 1+\cos \left[\mathbf{q} \cdot\left(\mathbf{r}_{1}-\mathbf{r}_{2}\right)+\chi\left(\mathbf{p}_{1}, \mathbf{r}_{1}\right)\right. \\
& \left.+\chi\left(\mathbf{p}_{2}, \mathbf{r}_{2}\right)-\chi\left(\mathbf{p}_{2}, \mathbf{r}_{1}\right)-\chi\left(\mathbf{p}_{1}, \mathbf{r}_{2}\right)\right] .
\end{aligned}
$$

Note that $\chi(\mathbf{p}, \mathbf{r})$ of Eq. (5) diverges logarithmically [17], but Eq. (6) converges at least as fast as $\left|\mathbf{r}_{1}-\mathbf{r}_{2}\right| /\left|\mathbf{r}_{1}+\mathbf{r}_{2}\right|$ because of the cancellation between $\chi$ 's. Numerically we find, for example, that the contribution beyond $15 \mathrm{fm}$ is negligible for the source size less than $8 \mathrm{fm}$.

Let us now consider the special case where the bosons emitted with parallel momenta. The apparent size of a spherically symmetric charged source can then be expressed as

$$
R_{\mathrm{app}}^{2}(P)=R_{0}^{2}+\frac{m}{P^{2}} T+\left(\frac{m}{P^{2}}\right)^{2} U^{2} .
$$

Here, $T$ and $U$ depend only on the source distribution and potential

$$
\begin{gathered}
T=\int d \mathbf{r}_{1} d^{3} \mathbf{r}_{2} g\left(\mathbf{r}_{1}\right) g\left(\mathbf{r}_{2}\right)\left(\mathbf{r}_{12} \cdot \hat{\mathbf{z}}\right)\left[I\left(\mathbf{r}_{1}\right)-I\left(\mathbf{r}_{2}\right)\right], \\
U^{2}=4 \int d \mathbf{r}_{1} d^{3} \mathbf{r}_{2} g\left(\mathbf{r}_{1}\right) g\left(\mathbf{r}_{2}\right)\left[I\left(\mathbf{r}_{1}\right)-I\left(\mathbf{r}_{2}\right)\right]^{2},
\end{gathered}
$$

where $I(\mathbf{r})=-v \chi(\mathbf{p}, \mathbf{r})$ is a function of the emission point, r. A close examination of Eqs. (7)-(9) shows [18] that the crucial quantity is essentially the ratio of the Coulomb and kinetic energies

$$
\zeta=\frac{Z e^{2}}{R_{0}} / \frac{P^{2}}{2 m},
$$

which becomes large for large $Z$ and low $P$.

The $T$ term in Eq. (7) is a linear functional of the Coulomb potential between the source and the emitted pair, and thus either increases or decreases $R_{\text {app }}$, depending on the emitted pair either negatively or positively charged. The $U$ term is, however, quadratic in the Coulomb potential and increases $R_{\text {app }}$ in all cases. For the cases considered below, $|\zeta|$ is significant when $P$ is smaller than a few hundred $\mathrm{MeV} / c$, or even less. The $U$ term can then contribute appreciably to $R_{\text {app }}$, and actually become dominant for a relatively wide range of small $P . R_{\text {app }}$ rises very rapidly $\left(\sim P^{-4}\right)$ for the low $P$. A contribution similar to the $T$ term is included, for example, in the discussion of Ref. [13], but a contribution similar to the $U$ term is not.

Equations (7)-(9) show that for a given $P$, the effect is stronger for heavier particles, for example, kaons in comparison to pions. The parameters $T$ and $U$ are proportional to the source charge in the eikonal approximation, and depend to a small extent on the source size and geometry. The exact expression of $R_{\text {app }}$ for arbitrary $\mathbf{P}$ and $\mathbf{q}$ is more complicated than Eq. (7). Numerical calculations show, however, that these observations obtained for the special case of the eikonal approximation still hold, and that Eq. (7) yields a quite good representation of the $P$ dependence of $R_{\text {app }}$ if we treat $T$ and $U$ as adjustable parameters. In this case, $T$ and $U$ follow semiquantitatively these observations, including rising with increasing source charge roughly linearly. In detail, $U$ representing the exact results (i.e., using exact wave functions) is smaller than those obtained with the eikonal wave function, especially for a large source charge. This is because $U$ is determined dominantly in the small $P$ region, where the eikonal wave function tends to overestimate the phase shifts. 


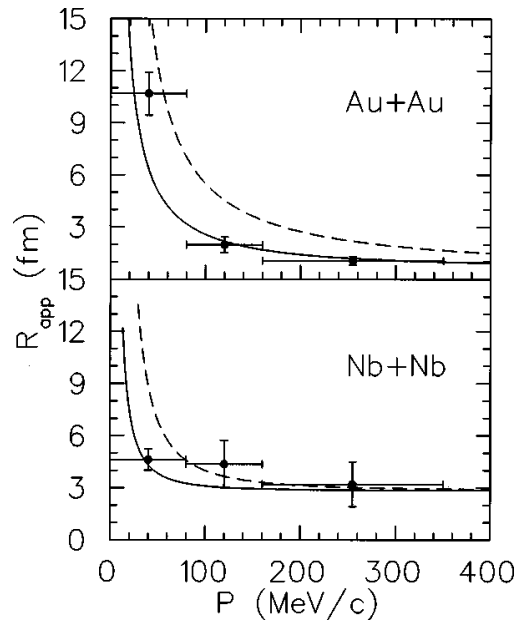

FIG. 2. The apparent source sizes from $\pi^{+} \pi^{+}$interferometry of $\mathrm{Au}+\mathrm{Au}$ (upper panel) and $\mathrm{Nb}+\mathrm{Nb}$ (lower panel) collisions at 650 $\mathrm{MeV} /$ nucleon, binned according to the total momentum $P$. The horizontal error bars of the data [19] represent the binning according to total momentum. The solid line represents the calculation obtained by numerically solving the Schrödinger equation [and by being smoothed by means of Eq. (7)] for $Z=158$ (upper panel) and for $Z=82$ (lower panel). The dashed lines are the similar calculation for $K^{+} K^{+}$pairs.

However, $T$ is insensitive to small $P$ and is calculated reasonably reliably by the eikonal approximation.

More importantly, Fig. 1 suggests that the effect of the source-pair interaction can be seen clearly when the data are binned according to the total momentum of the pair $P$. Though yet relatively crude, such data are available. Figure 2 show comparison of our exact calculation with data at 650 $\mathrm{MeV} /$ nucleon [19] for the positive boson pairs, $\pi^{+} \pi^{+}$and $K^{+} K^{+}$. For the sake of clarity, we show the calculation in Figs. 2 and 3 after smoothing out by means of Eq. (7) the curves obtained by numerical integration as those in Fig. 1. In the upper panel of Fig. 2, the calculated effect of a $Z$ $=158$ source charge is compared with $R_{\text {app }}$ extracted from the $\pi^{+} \pi^{+}$data of Au+Au central collisions, binned according to $P$ of the pair. We see that the calculation with the (true) size of $R_{0}=1.0 \mathrm{fm}$ reproduces the data well. We emphasize that $R_{0}$ is the only adjustable parameter in our model. The small value of $R_{0}$ is merely a reflection of the measured radii for the $\mathrm{Au}+\mathrm{Au}$ collisions [19]. In the lower panel of Fig. 2, the similar calculated effect of a $Z=82$ source charge with $R_{0}=2.8 \mathrm{fm}$ is compared with the $\mathrm{Nb}$ $+\mathrm{Nb}$ collision data. The agreement between our calculation and the data is also good.

As Fig. 2 explicitly shows, the source Coulomb effect is prominent for a small $P$ and is expected to be more significant in low-energy than high-energy collisions. In order to clarify this point, we make similar comparison at a higher energy. Figure 3 shows comparison for $\pi^{-} \pi^{-}$pairs at 1.8 $\mathrm{GeV} /$ nucleon. In the upper panel, the calculation for a $Z$ $=100$ source with $R_{0}=2.8 \mathrm{fm}$ is compared with the $\mathrm{Ar}$ $+\mathrm{Pb}$ collision data [21]; and in the lower panel, the calculation for a $Z=36$ source with $R_{0}=4.2 \mathrm{fm}$ is compared with the $\mathrm{Ar}+\mathrm{KCl}$ collision data [20]. While the agreement seems

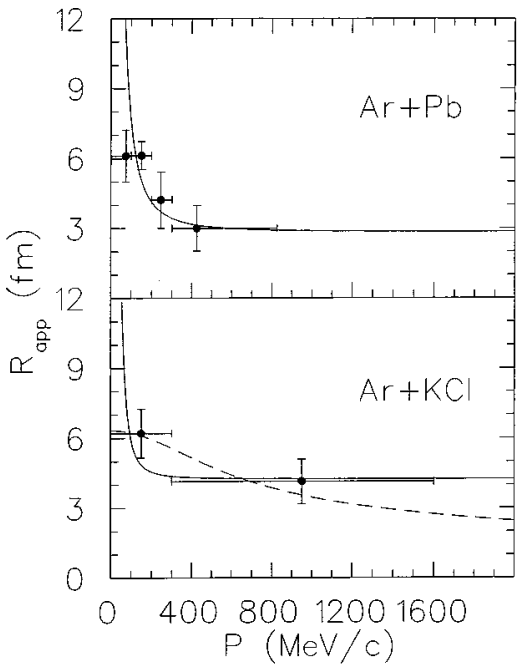

FIG. 3. The apparent source sizes from $\pi^{-} \pi^{-}$interferometry of $\mathrm{Ar}+\mathrm{Pb}$ (upper panel) and $\mathrm{Ar}+\mathrm{KCl}$ (lower panel) collisions at 1.5 $\mathrm{GeV} /$ nucleon, binned according to the total momentum $P$. Data of Refs. [21] and [20] in the upper and lower panels, respectively, show bin sizes. The calculation shown with the solid lines is by the numerical integration as in Fig. 2. The exploding source fit of Ref. [22] is shown by the dotted line in the lower panel.

to be reasonable, the source-Coulomb effect is significant in the limited low-momentum region of $P<100 \mathrm{MeV} / c$, as it is in Fig. 2. In a typical high-energy collision most pions will have momentum above this and not be affected by the source-Coulomb effect, so the only way of seeing the effect will be to specifically bin the data for low-momentum pions.

The extraction of the source size is a complicated issue, especially when the expansion of the source size is taken into consideration [6]. As a simple comparison, we also show in Fig. 3 a fit by an exploding source model [22]. This model neglects the charge of the source but does take into account a radial expansion of the source. Figure 3 shows that the model and the Coulomb source effect predict the same trend of larger sources for small total momentum pairs, but with different momentum dependence. This difference would allow the two effects to be disentangled, if experimental data are finely binned by total pair momentum. At any rate, both must be taken into account upon a realistic extraction of the source size from experimental data.

In our calculations, the only adjustable parameter $R_{0}$ is taken to be the asymptotic value of $R_{\text {app }}$ at large $P$. Note that the rise of the apparent size at small $P$ is determined by the charge of the source, the mass and charge of the outgoing particles, and weakly depends on $R_{0}$.

In summary, we have demonstrated quantum mechanically that the low-energy Coulomb interactions with the source charge increase the apparent source size for both of positive and negative charged boson pairs, and have presented a simple expression to account for the effect.

This work was supported by the National Science Foundation grant at Caltech (Grants No. PHY-9412818 and PHY9420470), and by the U. S. Department of Energy grant at CSUN (Grant No. DE-FG03-87ER40347). 
[1] S. Pratt, T. Csörgö, and J. Zimányi, Phys. Rev. C 42, 2646 (1990).

[2] M. Gyulassy, S. K. Kauffmann, and L. W. Wilson, Phys. Rev. C 20, 2267 (1979).

[3] S. Pratt, Phys. Rev. D 72, 72 (1986).

[4] M. G. Bowler, Phys. Lett. B 270, 69 (1991).

[5] M. Biyazima, T. Mizoguchi, T. Osada, and G. Wilk, Phys. Lett. B 353, 340 (1995).

[6] Yu. M. Sinyukov, R. Lednicky, S. V. Akkelin, J. Pluta, and B. Erazmus, Phys. Lett. B 432, 248 (1998).

[7] R. Lednicky and V. L. Lyuboshitz, Sov. J. Nucl. Phys. 35, 770 (1982).

[8] R. Lednicky, Nukleonika 43, 345 (1998).

[9] D. J. Dean and S. E. Koonin, Phys. Lett. B 305, 5 (1993).

[10] S. E. Koonin, Phys. Lett. 70B, 43 (1977).

[11] M. C. Chu, S. Gardner, T. Matsui, and R. Seki, Phys. Rev. C 50, 3079 (1994).
[12] B. Erazmus et al., Nucl. Phys. A583, 395 (1995).

[13] G. Baym and P. Braun-Munzinger, Nucl. Phys. A610, 286c (1996).

[14] L. Martin, C. K. Gelbke, B. Erazmus, and R. Lednicky, Nucl. Phys. A604, 69 (1996).

[15] D. Hardtke and T. J. Humanic, Phys. Rev. C 57, 3314 (1998); H. W. Barz, J. P. Bondorf, J. J. Gaardhoje, and H. Heiselberg, nucl-th/9711064.

[16] H. W. Barz, Phys. Rev. C 53, 2536 (1996).

[17] R. J. Glauber, in Lectures in Theoretical Physics, edited by W. E. Brittin and D. G. Dunham (Interscience, New York, 1959), Vol. 1, p. 315.

[18] T. D. Shoppa, Ph.D thesis, Caltech, 1996.

[19] R. Bock et al., Mod. Phys. Lett. A 3, 1745 (1988).

[20] D. Beavis et al., Phys. Rev. C 27, 910 (1983).

[21] D. Beavis et al., Phys. Rev. C 34, 757 (1986).

[22] S. Pratt, Phys. Rev. Lett. 53, 1219 (1984). 